\title{
OPINION
}

\section{Welcoming students to the mathematics community: obstacles to "belonging"}

Noel-Ann Bradshaw, School of Computing and Digital Media, London Metropolitan University, London, UK. Email: N.Bradshaw@londonmet.ac.uk. Pronouns she/her

Tony Mann, School of Computing and Mathematical Sciences, University of Greenwich, London, UK. Email: A.Mann@gre.ac.uk. Pronouns he/him

\section{Abstract}

This paper reflects on some of the obstacles which lead some students, particularly those from nontraditional academic backgrounds, to question whether they "belong" to the mathematics community.

Keywords: Retention, transition, student success, belonging, community, barriers, attainment gap.

\section{Introduction}

"I loved mathematics and I was good at mathematics; I was also certain I did not have what it took to be a mathematician." Hottinger (2016) recalls her feelings as a woman studying mathematics who chose not to apply for graduate studies in the discipline. Many mathematics students experience similar doubts as to whether they really belong in the mathematical word.

Undergraduates studying mathematics degrees are joining the mathematical community. Like all communities, ours has its own language and conventions, and for some undergraduates, unfamiliarity with these may lead them to feel that they do not belong in the community. Some aspects of the history of the subject, if not addressed by their teachers, may also lead some potential mathematicians to feel unwelcome. This is particularly so for students from non-traditional academic backgrounds.

We discuss some of these potential barriers and how higher education mathematics teachers can help students come to feel that they belong.

This paper has been heavily influenced by two valuable short articles on "mathematical microaggressions" by Francis Su, who at the time was President of the Mathematical Association of America (Su, 2015 and Su, 2016).

\section{Potential barriers}

One barrier to a feeling of belonging to a community is the feeling that "people like me" do not belong to the community. The two authors of this paper had different feelings when they enrolled on their mathematics degrees.

When Tony arrived at university to study mathematics, he did not doubt his right to be there. The following factors helped.

- He was white, male, middle class and was joining straight from school

- Both his parents had been to university (and all his siblings would do so too)

- He had been to a school from which over $95 \%$ of the students went to university 
- Two schoolfriends were starting on the same course at the same university

- A very famous mathematician of the time had been a former pupil of his school

- There was a history of students from his school studying mathematics at this university

- Tony's school maths teacher had recently studied at that university and had introduced the relevant culture (in contrast, many school students today are taught mathematics by teachers who have studied other subjects at university)

- He had read traditional mathematics books and was familiar with traditional mathematical writing styles

But for many new mathematics students today, few of these factors may apply.

In contrast, Noel-Ann started her undergraduate degree as a mature student in her late 30's.

- Despite having attended a 'good' school she had not done well at A-level and was convinced she would be the worst student on the degree.

- She was worried that all the students would be school leavers and so she would not fit in.

- She had not done any formal mathematics for about 20 years.

Recalling his own feelings as a new student, Tony was taken aback by a recent conversation with some students. As a measure to help new students settle at university in the first few weeks of their first year, selected current students were assigned to mentor groups of new students. At a training session for these mentors, they were asked to describe how they had felt on arrival at university. These were successful students who were confident enough to volunteer to be mentors, and Tony expected that they would recall feelings of excitement, tinged with anxiety. However one word dominated: that word was "terrified". The strength of this feeling, and that it was shared by all the students in the group, shocked and dismayed Tony. Tutors need to be aware just how uncertain many - perhaps most - new students feel about their arrival at university, and work to foster the sense that students belong in their new environment. Initiatives such as the Maths Arcade and mentoring schemes for new students are relevant here (Bradshaw 2011, Bradshaw 2017, Rowlett et al 2019, Grove et al 2015).

This paper focuses particularly on the subject-specific feeling of belonging and will discuss some barriers which may cause students to feel that they are not members of the mathematical community. For a very recent discussion of the challenges facing some mathematicians see Crowell (2021).

\section{1. (Lack of) role models}

"I get a lot of letters from young queer scientists. They get hope for their professional futures just knowing I exist. Imagine that. Imagine not knowing anyone like you in your field. Imagine not even hearing of anyone like you" (Kent, 2019).

As mentioned above, Tony was aware of many examples of "people like him" studying mathematics at his university and had no reason to doubt his right to be there. On the other hand, Noel-Ann has no recollection of any role models for mature women students. The visibility of diverse role models can reassure students that "people like them" belong to the mathematics community. Examples of initiatives to provide such role models and other resources include: 
- The book Living Proof: Stories of Resilience Along the Mathematical Journey, published by the American Mathematical Society and the Mathematical Association of America and available as a free ebook (Henrich et al, 2019)

- Irina Linke and Eugenie Hunsicker's video "Faces of Women in Mathematics" (Linke and Hunsicker, 2018)

- The "Mathematicians of the African Diaspora of the Twentieth Century" website (Williams, 2021)

- The website "Mathematically Gifted \& Black", "Featuring the Accomplishments of Black Scholars in the Mathematical Sciences" (The Network of Minorities in Mathematical Sciences, 2021)

- Nira Chamberlain's presentations on "The Black heroes of mathematics" (eg Chamberlain, 2020)

- The website of Lathisms, which presents "Latinxs and Hispanics in the Mathematical Sciences" (Lathisms, 2021)

- Profiles on the Institute of Mathematics and its Applications (IMA) "Maths Careers" website (IMA, 2021a)

- The IMA's "Diversity and Inclusivity" web page, which at the time of writing links to pages on BAME (Black, Asian and Minority Ethnic), Disability and Neurodiversity, Gender Diversity, LGBTQ+, and Women in Mathematics. (IMA, 2021b).

- The London Mathematical Society (LMS) "Success Stories” website (LMS, 2021)

- Chalkdust magazine's Black History Month (Chalkdust, 2019)

Such resources are potentially valuable in helping undergraduates from different backgrounds feel the community of mathematicians does contain people like themselves.

\section{2. (Lack of) inclusivity in examples}

It is not only the absence of role models that can lead some to feel that the mathematics community does not include people like them. Students learning mathematics will come across many examples illustrating applications of the material they are learning. These examples may refer to imaginary people. Intuitively, it might seem that vivid examples help in learning mathematics, and concrete examples are often more helpful than abstract ones, so it is natural to give names to the people who feature in examples or exam questions. (However the value of concrete rather than abstract examples in learning mathematics has been challenged, for example by De Bock et al (2011).)

If your name is Mary or Ahmed, for example, and if the problems you meet in your mathematics exercises always refer to people called Michael, Peter, and William, then you may infer a message that people like you are not expected to study mathematics. So, to make our students feel welcome, we should strive to make sure that the names we use in our examples reflect diversity in gender and cultural background. However, the question of names is complicated, not least because of the sensitivity we as human beings have about our names and our cultures, and because we are not confident about using names from other cultures. Possible approaches include 
- Avoiding using names completely (which means abandoning a tool which helps students engage)

- Using names which might come from different genders and cultures, like "Ali" and "Lee"

- Using names drawn from previous students from different cultures

- Use random name generators which draw names from a representative database

Each of these approaches has its limitations!

It is not only names which can exclude students. If you identify as LGBTQ+ and a mathematics class presents a "marriage theorem" in which men are paired with women, or if you are non-binary, and a logic class presents "gender" as a binary property, then you may feel excluded. (It is not surprising, perhaps, that there are different views on these issues. An Australian lecturer's attempt to discourage the use of the word "marriage" in discussing matching theorems led to press reports of a hostile reaction from at least one student (SBS News, 2017).)

In creating examples to illustrate mathematical ideas, we tend to choose settings with which we are familiar. But our background is often different from that of our students, and these settings may not help their sense of belonging. People do not all have exactly one job, children may not have piano lessons or study ballet, and summer holidays and going to the theatre may be unfamiliar concepts for some of our students. Examples about football or television programmes may engage some students but alienate others. References to music or films may not be understood by young people from different backgrounds, and, more importantly than not understanding, they may feel that they are in some way at fault because they don't meet tutors' expectations. Humour can be particularly problematic for the student who does not "get" the joke, but humour is also a valuable tool in engaging students and building a relationship with them.

An example discussed by Su (2015) is the well-known Fermi problem of estimating how many pianotuners there are in the UK. Tony has used this with students, aiming to boost their confidence by showing that they can come up with a good estimate for a problem they haven't previously considered. But students may not know what a piano-tuner does, or may assume that, like a guitarist, a serious pianist tunes their own piano. Since the value of the exercise is to show students that they can solve unfamiliar problems, is that a problem? Well, it is a problem if the students think that they are expected to know all about piano-tuners in advance. So it is important to be clear to students about the purpose of the exercise and that there is no expectation that the problem will be familiar to them.

There are examples of mathematical results (such as the marriage theorem mentioned earlier) which are traditionally presented in contexts which, today, would be considered inappropriate by some. Another example is the "Battle of the Sexes" in game theory (Wikipedia, 2021). In the original formulation, this posts that a man and a woman hope to meet at either a prize fight (preferred by the man) or a ballet (preferred by the woman). They cannot communicate: which should each go to if they want to be at the same event as the other? (Apparently, some recast the problem as "Bach or Stravinsky" rather than "fight or ballet". It seems unlikely that that would make the example more relevant to most of today's students.) Of course, quite apart from the gendered assumptions about preferences, in the days of mobile phones it might be hard to persuade students that this is a reallife problem!

This raises a question. These problems, and their traditional formulations, are part of the culture of mathematics. They can be found in standard textbooks, some of which might be recommended 
reading for students. When they were created, there was an element of humour: these were not proposed uncritically as realistic real-life problems. To what extent should we, in aiming to be inclusive, avoid referencing past examples which are now problematic? Are we helping students to belong, or in fact, by denying them access to traditional mathematical presentations, even perhaps achieving the unwanted result of excluding them from mathematical culture?

While there is no simple solution, awareness of the potential issues, understanding that one's own cultural context may not match that of students, and an attempt to use diverse examples and references, may help reassure students that they are not being intentionally excluded.

\subsection{Mathematical language and notation}

The culture of mathematics includes use of language in ways which are sometimes subtly different from standard English usage. This may confuse students who are not familiar with the nuances of mathematical discourse, and lead them question their ability to study the subject.

For example, the words "infinite" and "unlimited", in everyday language, are often used just to mean "very large", while in mathematics they have more precise meanings. Similarly, to many laypeople, "theory" suggests an unproven hypothesis, whereas in mathematics terms like "group theory" refer to an established body of proven results. The term "proof" also has somewhat different meanings in mathematics and everyday life.

A few years ago Tony saw a mobile phone contract advertisement offering "unlimited data" which, in the terms and conditions, was very specifically limited to something like 5GB per month. He was sufficiently irritated by the use of "unlimited" to describe something with a very specific limit that he wrote to the Advertising Standards Authority, whose reply indicated that there was no problem because "unlimited" simply means "very large" and does not imply that there is no limit. Rightly or wrongly, that is the usage with which students are likely to be familiar.

Particularly sensitive is the mathematical use of words like "trivial" and "obvious". There is the old joke about the mathematician who says in a lecture "this result is obvious", then hesitates, spends several minutes considering, concludes "I am not sure this is obvious", and then after further thought comes back the next day and says "Yes, this is obvious". The point is that when mathematicians use the word "obvious" it does not imply that you should be able immediately to see why the result is true - it may take some thought. But for a student unfamiliar with this use, they may feel "I don't see why this is true therefore I am not good enough to be a mathematician". The word "trivial" has similar negative connotations in everyday language - Tony remembers an occasion when a leading mathematician was berated on an online history of mathematics forum when they used "trivial" to describe the proof of a mathematical result and some readers found the use of the word insulting. These examples show the sensitivities around the use of language, and indeed variations in different mathematical cultures.

Notation may also present challenges for students. For example, mathematicians make frequent use of Greek letters. Whereas Tony had studied ancient Greek at school and had no problem with this, students today are unlikely to have this advantage. Indeed, for many English will be a second or third language, and for some the Latin alphabet will not be used in their native language. (Tony recalls struggling with a textbook which used old German capital letters to denote algebraic structures: he could not tell them apart, and didn't have the sense to practice writing them himself until they became familiar.) While students may need to gain familiarity with Greek letters in order to follow mathematical expositions, teachers should appreciate that they may not arrive at university with this facility. 
Students may not have a full understanding of mathematical terminology and may not appreciate the distinctive features of a "theorem", a "lemma", a "corollary" and other technical terms. While part of the process of becoming a mathematician is to come to understand these terms through experience - through seeing them in action - in addition to learning from definitions - once again tutors might think about how they can help students develop their understanding of mathematical language.

So these examples - using words in a mathematical sense, or using symbols that may be new to students - might lead students unfamiliar with the practices to feel that they don't belong. But on the other hand belonging to the community requires understanding these usages and therefore it is important that students be exposed to mathematicians' use of language.

There is tension here - we want to help students belong to the mathematical community, and to understand the nuances and conventions of mathematical language and notation, but these can be a barrier for those new to the community. So tutors need to be sensitive to the potential difficulties students might have, and offer students support in learning how mathematicians communicate. They should consider whether it is necessary to explain the mathematical use of terms like "unlimited" and "obvious" when they use these words in their classes, and should consider whether students will have met the notation and symbols they use. While study of traditional mathematical textbooks will help students appreciate the standard language of the discipline, students increasingly turn to online resources and videos to support their learning, and these may not present such clear models. Bradshaw and Richardson (2013) describe an initiative to encourage mathematics students to read books about the subject - many (though not all) popular mathematics books do show how "insiders" express mathematical ideas.

As with other issues discussed in this paper, there are not always easy solutions, but if tutors are aware of the potential for students to feel excluded, their sensitivity may mitigate the adverse effects.

\subsection{The difficulty of mathematics}

University mathematics can be difficult. Practising mathematicians do not find their work easy. New ideas take time to assimilate: as John von Neumann famously said to a friend, "Young man, in mathematics you don't understand things. You just get used to them." (Wikiquote, 2021). Most of us are familiar with difficult mathematical ideas which took us days, weeks or even years to fully understand.

But this has not been the experience of some of our students. For those to whom school mathematics came easily, meeting mathematical ideas which they don't immediately grasp is a new experience, and one that can cause them to wonder whether they are good enough to succeed in the subject.

So it is important to prepare students for the experience of coming across deep ideas that require time to appreciate. We need to be clear that University-level mathematics is challenging and that students will at times, and perhaps frequently, experience the frustration of being "stuck", and that this does not in any way mean that they are inadequate, or not fit to be mathematicians. References to the struggles of famous mathematicians (like Andrew Wiles's seven years of working on Fermat's Last Theorem) or to published accounts of their work (Villani, 2015) may help. Suggestions of strategies for dealing with being stuck may not only be practically useful but will reassure students appreciate that this is a normal part of doing mathematics. 
Of course, there is a balance between warning students of the demands of the subject, and putting them off by over-emphasising the difficulties! But it is important to be aware that the difficulty of the subject may cause some students to doubt whether they really belong.

\section{3. "Decolonising the curriculum"}

The topic of decolonising the curriculum is currently being debated in universities which are anxious to eliminate the attainment gap between students of colour and their white peers. This concept may be controversial, with some departments being unsure how it is relevant to the teaching of an abstract subject like mathematics. Rather than defining the term here, we intend, for the purpose of discussion in this section, to discuss more broadly how we might avoid our mathematics curricula and teaching creating an unwelcoming environment for some potential mathematicians - an objective which we believe all mathematics teachers would support.

This topic is important for many reasons, but this current paper will suggest only that teachers might consider whether the current curriculum, in this regard, might in some ways present obstacles to "belonging" for some students of colour, or other groups, making them feel uncomfortable or unwelcome. There are of course other reasons why this aspect of the curriculum is an important concern, but these are not the point of this article.

Do our mathematical curricula take the opportunities available to present mathematical ideas from different cultures? For example, pure mathematicians see the concept of proof as central to mathematics. The traditional view that proof originated in ancient Greece has been challenged, for example in the essays by many authors collected by Chemla (2012), which show that Mesopotamian, Chinese and Indian knew how to demonstrate the correctness of their methods. How far might we broaden our curriculum to inspire students by celebrating the diverse origins of our subject and the mathematics of different cultures.

Sometimes there might be easy "wins". For example, Tony has been teaching a module on codes and cryptography. This began by presenting historical examples of codes, such as semaphore and Morse code (which Tony remembered from Boy Scout books, but which are probably less familiar to today's students). But examples from other cultures - African drum communications, native American smoke signals - are not only equally relevant, but positively enrich the module and demonstrate the universality of the concepts being introduced. So a minor tweak to the module content, in a situation like this, can have immediate benefit.

There are other issues arising from the history of mathematics. Recently the statistical community and the Royal Statistical Society (RSS) have confronted the fact that several of the pioneers of mathematical statistics in the last century were involved in the eugenics movement and expressed racist or anti-Semitic views (Langkjær-Bain, 2019; RSS, 2019). Buildings, lecture theatres and prizes previously named after Karl Pearson (1857-1936), Francis Galton (1822-1911) and Ronald Fisher (1890-1962) have been denamed. (UCL, 2020; Rothamsted Research, 2020; Committee of Presidents of Statistical Societies, 2020; Gonville and Caius, 2020).

The UCL statement about its denaming of the former Pearson Building and Pearson and Galton Lecture Theatres quotes its then Provost, Professor Michael Arthur, who said "Although UCL is a very different place than it was in the 19th century, any suggestion that we celebrate these ideas or the figures behind them creates an unwelcoming environment for many in our community." This captures the threat posed to the sense of belonging by the apparent celebration of people who, although they may have contributed significantly to the advancement of science, expressed abhorrent views. 
In this paper we use Karl Pearson as an example, since he has been in the news regarding this issue: we are not suggesting that his actions are necessarily more reprehensible than those of many other mathematicians of the past. Several other examples of mathematicians whose words and actions are unacceptable today are discussed by Bingham (2020), while Fara (2021) shows that the science of Isaac Newton and the early scientific community of the Royal Society have uncomfortable connections with the slave trade. In light of current interest in these topics, it is likely that such cases will continue to feature in news stories.

How would you feel about learning about the mathematics of someone who described people like you as "inferior physically and mentally to the native population", as Pearson and Margaret Moul wrote about Jewish immigrants (Pearson and Moul, 1925)? (Of course, many readers may have personally experienced such situations.) There are anecdotal reports of students at a UK university objecting to a module taught using the Moore Method, the enquiry-based learning method associated with R.L. Moore (1882-1973), because of Moore's racism - he refused to teach Black students and walked out of a seminar when he realised the speaker was Black (Wikipedia 2021b). If your tutors are presenting, without any apparent uncomfortableness, the mathematics of those who considered people like you as inferior, do you feel that you are really welcome in the class? Where we are aware of racist or sexist context relating to the mathematics we are teaching, perhaps we owe it to our students to acknowledge this explicitly in our teaching.

How might students feel about using results named after mathematicians who behaved offensively? For example how does a student who is aware of Pearson's racism feel about using "Pearson's correlation coefficient"? How would any of us feel about using " $X$ 's Theorem" if $X$ were, say, a mass murderer or child abuser? (Of course, this problem is not new. Mathematicians refer, for example, to the Bieberbach Conjecture, named after Ludwig Bieberbach (1886-1982), who was an active Nazi, and to the Bloch Space and Bloch Constant, named after André Bloch (1893-1948), who murdered three members of his family (Wikipedia 2021c, 2021d). And it arises in other areas of life - how should we regard books, films or music written or performed by people responsible for reprehensible actions or writings?)

It can be argued that naming a mathematical result after a mathematician associated with it is simply an attribution and does not imply any celebration of any other aspect of that mathematician's life. As historians are aware, people are complex, change their views, say and write things they later regret, and are very much influenced by the culture of their time. Very few of us will avoid expressing opinions or carrying out actions which will seem reprehensible to posterity.

Bingham (2020) argues that we should stop using names of mathematicians and refer to results descriptively rather than using the creator's name. There have already been moves to rename some mathematical results. For example, "Travelling Salesperson Problem" is used instead of "Travelling Salesman Problem", and "Route Inspection Problem" instead of "Chinese Postman Problem" (Wikipedia 2021e, 2021f). So although renaming mathematical results might cause some confusion to students consulting older books, there are already precedents.

A workshop exploring this issue at the University of Greenwich, led by the second author and a mathematics graduate who had been President of the University's BAME (Black, Asian and Minority Ethnic) Society, found that students were divided about measures such as denaming mathematical results, with most feeling that there are complicated and potentially divisive issues with no simple solution. However they did feel that lecturers should provide context for the mathematics they are presenting. We could present Pearson's work, and credit him for his mathematics, while acknowledging the offensive aspects of his life. We could indicate the racist and sexist nature of the societies which produced the mathematics our students are learning. 
So one strategy for avoiding creating an "unwelcoming environment" for students would be, rather than hiding or ignoring the racist nature of important mathematicians and the cultures in which they lived and worked, openly to discuss the issues. We might explicitly acknowledge the flawed nature of the human beings who contributed to our subject and the regrettable aspects of their times, celebrating the mathematics while deprecating the unpleasant aspects of the cultures in which it was developed and the failings of its creators.

\section{Assessment}

Students may doubt their place in the mathematics community if they are assessed by methods which don't allow them to demonstrate their individual abilities. The question of assessment in mathematics is a topical one (MEI 2021), and many UK universities explicitly encourage the use of a range of assessment types in all disciplines. Although many academic disciplines at UK universities have moved away from traditional timed examinations, these still comprise a large part of assessment in mathematics at most universities in the UK, and this (regrettably, in the view of the authors of this paper) has, in many cases, not changed significantly over the last ten years (lannone and Simpson, 2021).

Students who have been well prepared for examinations at school and have achieved good results in previous exams may enjoy this form of assessment, and may dislike and distrust alternatives. Some international students trained under different educational systems may have no experience of other assessments and will not expect to be assessed in any other way (and may not appreciate UK conventions around plagiarism in coursework).

But on the other hand formal examinations can be intimidating. Many students are very apprehensive about taking exams and prefer being assessed in other ways. This may particularly apply to those who have been educated at less academic schools or who have studied school qualifications which are not primarily assessed by examination. Anecdotal evidence from applicants to university in 2021 suggests that the disrupted A-level experience for those taking the qualification in 2020 and 2021 has left many students very nervous about the prospect of university examinations.

Both authors of this paper have seen many examples of mathematically able students who, under the stress of formal examinations, were unable to perform to their potential in assessments which contributed significantly to their degree outcome. The authors have doubts as to whether examinations are a reliable form of assessment and whether they benefit students' and teachers' physical and mental health, and would argue for variety of assessment methods in mathematics degrees. (There is discussion of the merits of different forms of assessment for mathematics in MEI (2021).)

The question of assessment in mathematics is a large and important one, deserving fuller analysis and discussion than space permits here. In this context the authors simply wish to identify that the forms of assessment used in higher education may have a profound effect on students' self-belief as aspiring mathematicians.

\section{Conclusion}

The authors have discussed some of the reasons why students might question whether they belong in the mathematics community. These are complicated issues and the authors do not claim to have solutions. However awareness of the feelings of doubt that students might have, and the factors which might make them feel that they are outside, and perhaps not welcome in, the community, will help tutors to present mathematics inclusively and to encourage their students to think of themselves as genuine mathematicians. 


\section{Acknowledgements}

The authors are grateful to many colleagues and students whose views and experiences have helped them develop their appreciation of the issues discussed. They would particularly like to express their thanks to an anonymous referee for extremely helpful comments and suggestions, which have resulted in a greatly improved paper.

\section{References}

Bingham, A., 2020. The history is the history. AMS Graduate Student Blog. Available at https://blogs.ams.org/mathgradblog/2020/07/08/the-history-is-the-history/ [Accessed 9 August 2021]

Bradshaw, N. (2011) The University of Greenwich Maths Arcade. MSOR Connections, 11 (3). pp. 26-29.

Bradshaw, N., 2017. The Maths Arcade: A Tool for Supporting and Stretching Mathematics Undergraduates. In: Wood, L. and Breyer, Y. (eds.), Success in Higher Education: Transitions to, within and from University. Singapore: Springer Singapore, pp. 95-110.

Bradshaw, N. and Richardson, K., 2013. Book Reviews for Mathematics Undergraduates: an assignment to enhance reading and writing skills essential for today's workplace and to promote collaboration between library and academic staff. Compass: Journal of learning and teaching, Vol 4, no. 7 , University of Greenwich, available at https://journals.gre.ac.uk/index.php/compass/article/view/98 (Accessed 9 August 2021]

Chamberlain, N., 2020. The 2020 Pascal Lecture: The Black heroes of mathematics [video]. Available at https://www.youtube.com/watch?v=g5nyGtQVKnc [Accessed 9 August 2021]

Chalkdust, 2019. Black mathematician month. Available at https://chalkdustmagazine.com/blackmathematician-month/ [Accessed 9 August 2021]

Chemla, K., 2012. The History of Mathematical Proof in Ancient Traditions. Cambridge: Cambridge University Press

Committee of Presidents of Statistical Societies, 2020. COPSS statement on R A Fisher award. Available at: https://imstat.org/2020/07/16/copss-statement-on-r-a-fisher-award/ [Accessed 9 August 2021]

Crowell, R., 2021. Modern Mathematics Confronts Its White, Patriarchal Past: Mathematicians want to think their field is a meritocracy, but bias, harassment and exclusion persist. Scientific American. Available at https://www.scientificamerican.com/article/modern-mathematics-confronts-its-whitepatriarchal-past/ [Accessed 16 August 2021]

De Bock, D. et al. Abstract or Concrete Examples in Learning Mathematics? A Replication and Elaboration of Kaminski, Sloutsky, and Heckler's Study. Journal for Research in Mathematics Education, vol. 42, no. 2, 2011, pp. 109-126. JSTOR, available at https://www.jstor.org/stable/10.5951/jresematheduc.42.2.0109 [Accessed 9 August 2021]

Fara, P., 2021. Life after gravity: Isaac Newton's London Career. Oxford: Oxford University Press

Gonville and Caius, 2020. College Council decides to remove R.A. Fisher Window from Hall. Available at: https://www.cai.cam.ac.uk/news/college-council-decides-remove-ra-fisher-window-hall [Accessed 9 August 2021] 
Grove, M., Croft, T., Kyle, J. and Lawson, D. (eds), 2015. Transitions in undergraduate mathematics education. Birmingham: University of Birmingham

Henrich, A., Lawrence, E., Pons, M., and Taylor, D. (eds), 2019. Living Proof: Stories of Resilience Along the Mathematical Journey. Providence, Rhode Island: The American Mathematical Society and The Mathematical Association of America. Available at https://www.maa.org/press/ebooks/living-proof-stories-of-resilience-along-the-mathematicaljourney-2 [Accessed 9 August 2021]

Hottinger, S., 2016. Inventing the mathematician: gender, race, and our cultural understanding of mathematics. Albany: State University of New York Press

lannone, P. and Simpson, A., 2021. How we assess mathematics degrees: the summative assessment diet a decade on. In Teaching Mathematics and Its Applications. Available at https://academic.oup.com/teamat/advance-article-abstract/doi/10.1093/teamat/hrab007/6271028

[Accessed 9 August 2021]

IMA, 2021a. Maths Careers Website: profiles. https://www.mathscareers.org.uk/profiles/ [Accessed 9 August 2021]

IMA, 2021b. Diversity and Inclusivity. https://ima.org.uk/about-us/diversity-and-inclusivity/ [Accessed 9 August 2021]

Kent, A., 2019. 'Cold, Austere or Queer'. In Henrich, A., Lawrence, E., Pons, M., and Taylor, D. (eds), 2019. Living Proof: Stories of Resilience Along the Mathematical Journey. Providence, Rhode Island: The American Mathematical Society and The Mathematical Association of America, pp. 4042. Available at https://www.maa.org/press/ebooks/living-proof-stories-of-resilience-along-themathematical-journey-2 [Accessed 9 August 2021]

Langkjær-Bain, R., 2019. The troubling legacy of Francis Galton. Significance, 16, pp. 16-21. Available at https://rss.onlinelibrary.wiley.com/doi/10.1111/j.1740-9713.2019.01275.x [Accessed 9 August 2021]

Lathisms, 2021. Latinxs and Hispanics in the Mathematical Sciences. https://www.lathisms.org/ [Accessed 9 August 2021]

Linke, I. and Hunsicker, E., 2018. Faces of women in mathematics [video]. Available at https://vimeo.com/259039018 [Accessed 9 August 2021]

LMS, 2021. Success stories. https://www.Ims.ac.uk/careers/success-stories [Accessed 9 August 2021]

MEI, 2021. MEI discussion paper on assessment in mathematics. Available at https://mei.org.uk/archive?section=news\&page=archive\&newsid=795 [Accessed 9 August 2021]

Network of Minorities in Mathematical Sciences, 2021. Mathematically Gifted \& Black. https://mathematicallygiftedandblack.com/ [Accessed 9 August 2021]

Pearson, K. and Moul, M., 1925, The problem of alien immigration into Great Britain, illustrated by an examination of Russian and Polish Jewish children. Annals of Eugenics, 1, pp. 5-54. Available at https://onlinelibrary.wiley.com/doi/10.1111/1.1469-1809.1925.tb02037.x [Accessed 9 August 2021] 
Rothamsted Research, 2020. Statement on R A Fisher. Available at: https://www.rothamsted.ac.uk/news/statement-r-fisher [Accessed 9 August 2021]

Rowlett, P., Webster, K., Bradshaw, N., and Hind, J., 2019. Evaluation of the Maths Arcade initiative at five U.K. universities. The Mathematics Enthusiast, 16 (1). Available at https://scholarworks.umt.edu/tme/vol16/iss1/23/ [Accessed 9 August 2021]

RSS, 2019. RSS contributes to UCL inquiry into history of eugenics. Available at: https://www.rss.org.uk/news-publication/news-publications/2019/general-news-(1)/rss-contributesto-ucl-inquiry-into-history-of-eug/ [Accessed 9 August 2021]

SBS News, 2017. UNSW lecturer discouraged use of the term 'marriage' in mathematics theorem. https://www.sbs.com.au/news/unsw-lecturer-discouraged-use-of-the-term-marriage-in-

mathematics-theorem [Accessed 9 August 2021]

Su, F. 2015. Mathematical Microaggressions. MAA Focus: Newsmagazine of the Mathematical Association of America, 35(5), pp. 36-37. Available at http://digitaleditions. walsworthprintgroup.com/publication/?i=278032\&p=36 [Accessed 9 August 2021]

Su, F., 2016. President's Message: The Secret Mathematical Menu. MAA Focus: Newsmagazine of the Mathematical Association of America, 35(6), pp. 28-30. Available at http://digitaleditions. walsworthprintgroup.com/publication/?m=\&l=1\&i=285056\&p=28 [Accessed 9 August 2021]

UCL, 2020. UCL denames buildings named after eugenicists. Available at: https://www.ucl.ac.uk/news/2020/jun/ucl-denames-buildings-named-after-eugenicists [Accessed 9 August 2021]

Villani, C., 2015. Birth of a Theorem: a mathematical adventure. London: The Bodley Head

Wikipedia, 2021a. The Battle of the Sexes. https://en.wikipedia.org/wiki/Battle of the sexes (game theory) [Accessed 9 August 2021]

Wikipedia, 2021b. Robert Lee Moore. https://en.wikipedia.org/wiki/Robert Lee Moore [Accessed 9 August 2021]

Wikipedia, 2021c. Ludwig Bieberbach. https://en.wikipedia.org/wiki/Ludwig Bieberbach [Accessed 9 August 2021]

Wikipedia, 2021d. André Bloch (mathematician). https://en.wikipedia.org/wiki/André Bloch (mathematician) [Accessed 9 August 2021]

Wikipedia, 2021e. The Travelling Salesman Problem. https://en.wikipedia.org/wiki/Travelling salesman problem [Accessed 9 August 2021]

Wikipedia, 2021f. The Route Inspection Problem. https://en.wikipedia.org/wiki/Route inspection problem [Accessed 9 August 2021]

Wikiquote, 2021. John von Neumann. https://en.wikiquote.org/wiki/John von Neumann [Accessed 9 August 2021]

Williams, $\quad$ S., 2021. Mathematicians of the African Diaspora. http://www.math.buffalo.edu/mad/PEEPS/madprofiles.html [Accessed 9 August 2021] 